Recepción: 11 / 03 / 2019

Aceptación: 17/ 04 / 2019

Publicación: 05 / 06 / 2019

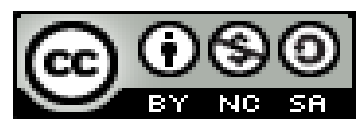

Ciencias técnicas y aplicadas

Artículo de investigación

\title{
Determinación de zonas susceptibles al fenómeno de desertificación en el cantón Ibarra, provincia de Imbabura
}
Determination of zones susceptible to the phenomenon of desertification in the Ibarra canton, province of Imbabura

Determinação de zonas suscetíveis ao fenômeno da desertificação no cantão de Ibarra, província de Imbabura

\author{
Paulina Gabriela Cascante-Almeida ${ }^{\text {I }}$ \\ ga_cascante@hotmail.com \\ Luis Bernardo Andrade-Muñoz II \\ andradebluis@gmail.com \\ Mauricio David Reyes-Pozo ${ }^{\text {III }}$ \\ nicozrs1@hotmail.com
}

Correspondencia: tga_cascante@hotmail.com

I. Especialista Superior en Gestión para la Reducción de Riesgos de Desastres, Ingeniera Agrónoma, Especialista Geomático en el Instituto Espacial Ecuatoriano, Quito, Ecuador.

II. Máster en Planificación Territorial y Gestión Ambiental, Ingeniero Agrónomo, Especialista Geomático en el Instituto Espacial Ecuatoriano, Quito, Ecuador.

III. Máster of Science Geographical Information Science\&Systems (UNIGIS M.Sc.), Ingeniero Agropecuario, Especialista Geomático en el Instituto Espacial Ecuatoriano, Quito, Ecuador. 
Determinación de zonas susceptibles al fenómeno de desertificación en el cantón Ibarra, provincia de Imbabura

\section{Resumen}

Este estudio se enfoca en la degradación de la tierra en zonas secas, en las cuales se produce el fenómeno de desertificación, uno de los problemas más serios de la región. Estudios realizados entre el Ministerio del Ambiente de Ecuador y el Ministerio de Agricultura y Ganadería determinaron que el $47 \%$ de los suelos de nuestro país se encuentran en procesos de degradación. Sin embargo, es un concepto desconocido para la mayoría o, al menos, no se es consciente de la dimensión del problema.

El objetivo de este trabajo es determinar las zonas susceptibles al fenómeno de la desertificación en el cantón Ibarra, a través del uso de los Sistemas de Información Geográfica (SIG) y la aplicación de la metodología desarrollada por la Agencia de los Estados Unidos para el Desarrollo Internacional, adaptada a la información de variables físicas (textura, pendiente) y climáticas (precipitación, evapotranspiración potencial) que se encuentran en nuestro país.

La zonificación realizada determinó que el 35 \% del área total del cantón (109 705,29 ha) tiene una susceptibilidad a la desertificación media a alta, esto se debe, principalmente, a la deforestación por el avance de la frontera agrícola y la utilización de técnicas intensivas en la agricultura; mientras que el $44 \%$ presentan baja susceptibilidad al fenómeno de la desertificación y únicamente el $21 \%$ de la superficie presenta susceptibilidad nula.

Palabras claves: desertificación; degradación; deforestación; sistemas de información geográfica.

\section{Abstract}

This study focuses on the degradation of the land in dry areas, in which the phenomenon of desertification occurs, one of the most serious problems in the region. Studies carried out between the Ministry of Environment of Ecuador and the Ministry of Agriculture and Livestock determined that $47 \%$ of the soils of our country are in degradation processes. However, it is an unknown concept for the majority or, at least, one is not aware of the dimension of the problem.

The objective of this work is to determine the zones susceptible to the phenomenon of desertification in the canton Ibarra, through the use of Geographic Information Systems (GIS) and the application of the methodology developed by the United States Agency for Development. International, adapted to the information of physical variables (texture, slope) and climatic (precipitation, potential evapotranspiration) that are found in our country. 
The zoning made determined that $35 \%$ of the total area of the canton (109 705.29 ha) has a susceptibility to medium to high desertification, this is mainly due to deforestation due to the advance of the agricultural frontier and the use of intensive techniques in agriculture; while $44 \%$ have low susceptibility to the phenomenon of desertification and only $21 \%$ of the surface has no susceptibility.

Keys words: desertification; degradation; deforestation; geographic information systems.

\section{Resumo}

Este estudo enfoca a degradação da terra em áreas secas, em que ocorre o fenômeno da desertificação, um dos problemas mais graves da região. Estudos realizados entre o Ministério do Meio Ambiente do Equador e o Ministério da Agricultura e Pecuária determinaram que 47\% dos solos de nosso país estão em processo de degradação. No entanto, é um conceito desconhecido para a maioria ou, pelo menos, um não está ciente da dimensão do problema.

O objetivo deste trabalho é determinar as zonas suscetíveis ao fenômeno da desertificação no cantão de Ibarra, através do uso de Sistemas de Informações Geográficas (SIG) e a aplicação da metodologia desenvolvida pela Agência de Desenvolvimento dos Estados Unidos. Internacional, adaptado à informação de variáveis físicas (textura, declive) e climáticas (precipitação, evapotranspiração potencial) que se encontram em nosso país.

Zoneamento realizada determinado que 35\% da área total do canto (109 705,29 ha) tem uma susceptibilidade a desertificação média elevada, isso é devido principalmente ao desmatamento avançando fronteira agrícola e usando técnicas intensivas em agricultura; enquanto $44 \%$ têm baixa suscetibilidade ao fenômeno da desertificação e apenas $21 \%$ da superfície não tem suscetibilidade.

Palavras chaves: desertificação; degradação; desmatamento; sistemas de informação geográfica.

\section{Introducción}

La desertificación es un proceso de degradación del territorio de zonas áridas, semiáridas y subhúmedas secas resultantes de diversos factores, tales como las variaciones climáticas y las actividades humanas, (Oropeza, 2007; Valderrama, 2017), interrelacionados, que se manifiestan a diferentes niveles Desertification is among the most serious problems in the region, studied carried out between the Ministry of the Environment of Ecuador and the Ministry of the Agriculture and Livestock, determinated that $47 \%$ our country's soils are in the process of being degraded. However, 
Determinación de zonas susceptibles al fenómeno de desertificación en el cantón Ibarra, provincia de Imbabura

it is an unknown concept for most or at least, you aren't aware of the size of the problem. The objective of this work is to determinate the susceptible zones to the phenomenonof the desertification Ibarra canton, through the use of Geographic Information Systems (SIG) and the application of the methodology developed by the United States Agency for International Development, adapted to the information if physical (texture, slope) and climatic variables (precipitation, potential evapotranspiration). The zoning made determined that $35 \%$ of the total area canton $(109705,29 \mathrm{ha})$ has to medium to high susceptibility to desertification due to the advance of the agriculture border and the use of the intensive techniques in agriculture, while $44 \%$ have low susceptibility the phenomenon of desertification and only $21 \%$ of the surface present no susceptibility.

de resolución tanto espaciales como temporales (García Ruiz et al., 1996).La comunidad internacional ha reconocido, desde hace tiempo, que la desertificación constituye un problema mayor de carácter económico, social y ambiental, que concierne a numerosos países en todas las regiones del mundo (COP6, 2003).

A nivel mundial, 1 de cada 5 personas vive en una zona afectada por desertificación. Una tercera parte de las tierras emergidas del planeta conforma las tierras secas. El mapa mundial de la desertificación (Naciones Unidas, 2018), advierte que esta amenaza se cierne sobre el $74 \%$ de Australia, el 34\% de África, el 31\% de Asia, el 19\% de América y el 2\% de Europa. Las actividades extractivas, como las industrias minera y petrolera, y la ganadería ovina extensiva han generado desertificación en una vasta área de América del Sur, puntualmente en la región patagónica (Paruelo y Aguiar, 2003).

Según el Programa de las Naciones Unidas para el medio Ambiente -PNUMA- (1991), en las tierras secas de América del Sur, según datos del problema de la desertificación alcanza cifras alarmantes: de 420,67 millones de ha (m. ha) de las tierras utilizadas para fines agrícolas 305,81 m. ha están degradadas, lo que representa un porcentaje del 72,7 \%. Entre éstas, las tierras cubiertas por pastizales, dedicadas a la producción animal (390,90 m. ha) son las que tienen mayor superficie afectada: 297,75 $\mathrm{m}$. ha, o sea un $76 \%$ del total. Le siguen las tierras de cultivo de secano $(21,35 \mathrm{~m}$. ha) de las cuales 6,64 m. ha están degradadas (31\%). Finalmente, de las 8,42 m. ha de las tierras de regadío 1,42 m. de ha están degradadas (17\%). Es evidente que revertir esta situación será no solamente muy difícil sino sobre todo muy costoso. Las principales medidas preventivas, correctivas o de rehabilitación tienen un alto costo por hectárea. La desertificación en tierras secas es uno de los mayores desafíos ambientales que enfrenta la sociedad humana (Oba, 2001; PNUMA, 2012). 
Así, la desertificación es el empobrecimiento y degradación de los geoecosistemas terrestres por sobreexplotación, uso y gestión inapropiados en territorios fragilizados por la aridez y las sequías (López, 2004; Ruiz y Febles 2004; Pulido y López, 2007). Cuando estos ecosistemas han sido sometidos a un uso abusivo de los recursos, pierden la posibilidad de recuperarse a presiones anteriores y pueden entrar en una espiral que conduce la desertificación (Abraham, Montana y Torres, 2006).

La Food Agriculture Organization -FAO- (1993) recomienda que todos los países -99 en total, según las cifras del PNUMA- en cuyo territorio haya tierras áridas, semiáridas y subhúmedas propensas a ser afectadas por la desertificación tomen conciencia de este fenómeno que repercute en la vida de 1 000 millones de seres humanos que viven en una superficie que abarca entre 3500 a 4000 millones de hectáreas, esto es, aproximadamente un 30 por ciento de las zonas continentales del planeta. López (2001) sostiene que la percepción del fenómeno varía de acuerdo a múltiples factores que van desde el grado de desarrollo hasta el conocimiento científico, cultural, económico y social de las poblaciones afectadas.

En Ecuador la desertificación se presenta con mayor intensidad en la zona costera, mientras que en la región interandina su impacto no es aún muy evidente, pero algunas provincias manifiestan una tendencia progresiva a la desertificación (Salomón et al., 2008). De acuerdo a los últimos estudios realizados entre el Ministerio del Ambiente de Ecuador (MAE) y el Ministerio de Agricultura y Ganadería (MAG), el $47 \%$ de los suelos de nuestro país se encuentran en procesos de degradación, siendo esta un proceso previo a la desertificación. Las provincias que presentan un mayor índice de procesos de desertificación son: El Oro, Loja, Azuay, Manabí, Chimborazo, Tungurahua, Guayas, Pichincha, Imbabura, Esmeraldas, Zamora-Chinchipe (MAE y MAG, 2017).

El Ecuador como país parte de la Convención de las Naciones Unidas de lucha contra la Desertificación (UNCCD), asumió el compromiso de presentar el Reporte Nacional sobre el estado de Desertificación, Degradación de la Tierra y Sequía (DDTS), así como las sinergias de la DDTS con el cambio climático, la diversidad biológica, seguridad alimentaria y otras afines (MAE y MAG, 2017). 

En el Plan Nacional para el Buen Vivir 2013-2017, uno de los lineamientos de la Estrategia Territorial Nacional es la de garantizar la protección de la población, los servicios sociales y básicos, las actividades económicas y la infraestructura vial, de apoyo a la producción y energética de los efectos de desastres naturales o socio naturales, mediante la ejecución de procesos de prevención y mitigación de riesgos (SENPLADES, 2013).

Según el análisis del sistema territorial actual del cantón Ibarra, el 64 \% de la superficie es apto para bosques, pero solo el $24,11 \%$ está ocupado por éste, lo que significa que se ha venido desarrollando un proceso de deforestación por el avance de la frontera agrícola en todo el cantón, dando como resultado procesos de desertificación, agudizado por las escazas precipitaciones que se registran en todo el año (GADM Ibarra, 2016).

La presente investigación tiene como propósito llegar a determinar las zonas susceptibles al fenómeno de la desertificación en el cantón Ibarra, mediante el análisis de las variables físicas y climáticas que influyen para su desarrollo; a través del uso de los SIG, generando información que podría ser utiliza en la planificación y toma de decisiones por parte de los encargados de la gestión de los recursos naturales en el cantón.

\section{Metodología}

La metodología aplicada toma como referencia el manual sobre el Manejo de Peligros Naturales en la Planificación para el Desarrollo Regional Integrado de la Agencia de los Estados Unidos para el Desarrollo Internacional (USAID) (OEA, 1993), para determinar las zonas susceptibles al fenómeno de la desertificación, mediante el uso de ciertas variables físicas (ej. textura del suelo) y climáticas (ej. precipitación).

\section{Recopilación de información}

Esta fase comprendió la revisión, análisis y evaluación de la información (base de datos) disponible sobre las temáticas de geomorfología (geoforma-pendiente) y edafología (clase textural del suelo), de la zona en estudio (Figura 1), levantada por el Instituto Espacial Ecuatoriano -IEE- en el año 2015, además del inventario nacional de todas las estaciones de la Red de Estaciones Meteorológicas Convencional 2017, del Instituto Nacional de Meteorología e Hidrología (INAMHI) del que se obtuvo 
los registros históricos de 10 años, sobre precipitación y evapotranspiración del cantón, para el cálculo de la relación $\mathrm{P} / \mathrm{EPT}$.

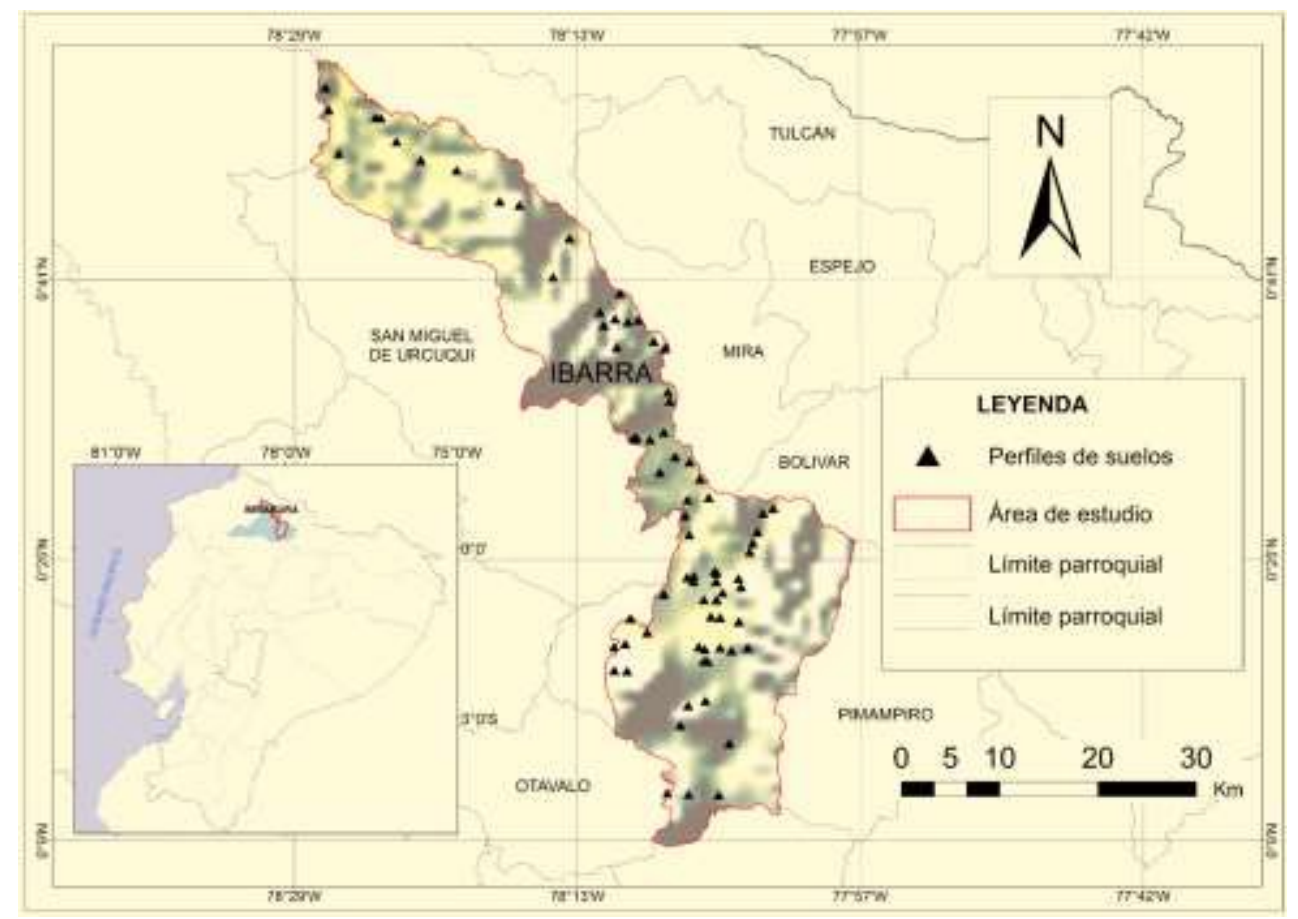

Figura 1. Ubicación del área de estudio y de los perfiles de suelos

Fuente: Elaboración propia

\section{Procesamiento y sistematización de los datos}

Mediante el uso del SIG, se llevó a cabo el procesamiento de la información para determinar las zonas susceptibles a éste fenómeno. Se generó una base de datos, con la información de las variables físicas (forma del relieve, pendiente, textura del suelo) y climáticas (precipitación, evapotranspiración y relación entre P/ETP), teniendo como base la unidad cartográfica -forma del relieve-. Para la obtención de las zonas susceptibles a desertificación se realizó el contraste de las capas suelopendiente y la relación precipitación/evapotranspiración, aplicando la metodología desarrollada por la USAID (OEA, 1993), y mediante el uso y aplicación de un Script (lenguaje de programación) que permitió automatizar y facilitar el procesamiento de la información al momento de determinar las áreas susceptibles a la desertificación.

Identificación del fenómeno de la desertificación 


\section{Caracterización climática}

- Precipitación

La información sobre la precipitación, se presenta en forma de isoyetas medias anuales en intervalos de $100 \mathrm{~mm}$ (en formato shapefile), a través de las cuales se determinaron las zonas con mayor ocurrencia de sequías, donde, de acuerdo a la metodología aplicada, las áreas con niveles de precipitación inferiores a $1500 \mathrm{~mm} /$ año son propensas al proceso de desertificación, debido a los periodos medios y largos de sequía que presentan.

\section{- Evapotranspiración}

La determinación de este parámetro depende de muchos factores ambientales -temperatura, radiación, viento, déficit de saturación, entre otros- (Valverde, 2007).Al igual que los datos de precipitación (isoyetas), se utilizó el archivo shapefile en donde se registra la información media de la evapotranspiración, así como los rangos de la misma.

\section{Relación precipitación-evapotranspiración}

Con los registros de precipitación y evapotranspiración, promedios mensuales, se procedió a calcular la relación entre estas dos variables, tomando como referencia la información del Cuadro1.

Cuadro 1. Clasificación de susceptibilidad según la precipitación y evapotranspiración potencial

\begin{tabular}{|c|c|}
\hline P/ETP & SUSCEPTIBILIDAD \\
\hline $0,01-0,75$ & Alta \\
\hline $0,75-1,0$ & Media \\
\hline$>1,0$ & Baja \\
\hline
\end{tabular}

Fuente: IEE-MAGAP, 2015

\section{Caracterización física}

- Textura del suelo

Empleando la información edafológica contenida en los perfiles de suelo levantados en el cantón por el IEE en el 2014 (Figura 1), se obtuvo la textura de los suelos, de las cuales se utilizó el porcentaje de arena (variable necesaria para el modelo de desertificación) de acuerdo a la clasificación americana (Cuadro 2). 
Determinación de zonas susceptibles al fenómeno de desertificación en el cantón Ibarra, provincia de Imbabura

Cuadro 2. Tipos de suelos y texturas de acuerdo a la clasificación americana

\begin{tabular}{|c|c|c|c|c|}
\hline \multirow{2}{*}{ Tipos de suelo } & \multirow{2}{*}{ Textura } & \multicolumn{3}{|c|}{ Relación (\%) } \\
\hline & & Arena & Limo & Arcilla \\
\hline \multirow{2}{*}{ Livianos } & Arenoso & 90 & 5 & 5 \\
\hline & Arenoso franco & 80 & 15 & 5 \\
\hline \multirow{4}{*}{ Medios } & Franco arenoso & 65 & 25 & 10 \\
\hline & Franco & 40 & 40 & 20 \\
\hline & Franco limoso & 20 & 65 & 15 \\
\hline & $\begin{array}{l}\text { Franco arcillo } \\
\text { arenoso }\end{array}$ & 35 & 35 & 30 \\
\hline \multirow{6}{*}{ Pesados } & Franco arcilloso & 35 & 30 & 35 \\
\hline & $\begin{array}{l}\text { Franco arcillo } \\
\text { limoso }\end{array}$ & 10 & 35 & 55 \\
\hline & Limoso & 10 & 85 & 5 \\
\hline & Arcillo arenoso & 55 & 5 & 40 \\
\hline & Arcillo limoso & 5 & 50 & 45 \\
\hline & Arcilloso & 20 & 20 & 60 \\
\hline
\end{tabular}

Fuente: USDA, 2006

- Pendiente

Información correspondiente al análisis cuantitativo/morfométrico del relieve, es decir al aspecto medible de la descripción de la morfología, levantada por el IEE en el cantón, y se refiere al grado de inclinación del terreno con relación a la horizontal; está expresado en porcentaje.

\section{Modelo para desertificación}

Para determinar las zonas susceptibles al fenómeno de la desertificación se empleó las variables de precipitación, relación precipitación/evapotranspiración, porcentaje de arena, pendiente de la forma del relieve, utilizando la clasificación que se muestra en el Cuadro 3. 
Cuadro 3. Modelo de susceptibilidad a desertificación

\begin{tabular}{|c|c|c|c|c|c|}
\hline Clase & $\begin{array}{c}\text { Precipitación } \\
\mathbf{( m m})\end{array}$ & $\mathbf{P} / \mathbf{E T P}$ & $\begin{array}{c}\text { Arena } \\
\mathbf{( \% )}\end{array}$ & $\begin{array}{c}\text { Pendiente } \\
\mathbf{( \% )}\end{array}$ & Susceptibilidad \\
\hline N & $>1500$ & & & & Nula \\
\hline B1 & $<1500$ & $>1$ & $>50$ & $>25$ & Baja \\
\hline B2 & $<1500$ & $>1$ & $<50$ & $<25$ & Baja \\
\hline B3 & $<1500$ & $>1$ & $<50$ & $<25$ & Baja \\
\hline B4 & $<1500$ & $>1$ & $<50$ & $<25$ & Baja \\
\hline M1 & $<1500$ & $0,75-1$ & $<50$ & $<25$ & Media \\
\hline M2 & $<1500$ & $0,75-1$ & $<50$ & $<25$ & Media \\
\hline M3 & $<1500$ & $0,75-1$ & $<50$ & $<25$ & Media \\
\hline M4 & $<1500$ & $0,75-1$ & $<50$ & $<25$ & Media \\
\hline A1 & $<1500$ & $0,01-0,75$ & $<50$ & $<25$ & Alta \\
\hline A2 & $<1500$ & $0,01-0,75$ & $<50$ & $<25$ & Alta \\
\hline A3 & $<1500$ & $0,01-0,75$ & $<50$ & $<25$ & Alta \\
\hline A4 & $<1500$ & $0,01-0,75$ & $<50$ & $<25$ & $<25$ \\
\hline
\end{tabular}

Fuente: OEA, 1993

Donde:

N: Precipitación media anual mayor a los $1500 \mathrm{~mm}$

Una precipitación media anual de más de $1500 \mathrm{~mm}$ generalmente indica un lugar situado en la zona de vida de bosques húmedos del sistema de clasificación de Holdridge (1967). En consecuencia, aún si la precipitación anual es cercana a $1500 \mathrm{~mm}$, su distribución en conjunto debe ser evaluada, dado que marcados períodos húmedos y secos podrían indicar problemas potenciales de desertificación en términos de pérdida de suelos por erosión del agua o del viento.

B1: Precipitación anual media $<1500 \mathrm{~mm}$; P/ETP 1,0 +; $>50 \%$ de arena; pendiente $>25 \%$.

La poca evapotranspiración comparada con el nivel de precipitación que reciben estas áreas indica que estas regiones tienen una temperatura relativamente baja. Si se acorta el período de crecimiento en las áreas más frías, ciertos tipos de agricultura y ganadería (intenso pastoreo, pisoteo, arado, terreno en descanso, y algunas actividades de construcción) eliminarían o reducirían la cobertura del terreno durante ciertas épocas del año.

B2: Precipitación anual media $<1500 \mathrm{~mm}$; P/ETP 1,0 +; $>50 \%$ de arena; pendiente $<25 \%$

Con estas características hay limitado potencial para casi todo tipo de desertificación, especialmente cerca del extremo superior del rango de precipitación y con relaciones P/ETP más altos. El principal peligro de desertificación sería el viento, este causaría problemas de erosión si la tierra fuera dejada 
Determinación de zonas susceptibles al fenómeno de desertificación en el cantón Ibarra, provincia de Imbabura

sin vegetación. Las temporadas de crecimiento de los cultivos generalmente serían cortas, y un descanso de terrenos sin cultivos coincidiría probablemente con el período de vientos.

B3: Precipitación anual media $<1500 \mathrm{~mm} ;$ P/ETP 1,0+; $<50 \%$ de arena; pendiente $>25 \%$

Este grupo presenta una relación P/ETP mayor de 1,0. Una relación alta de precipitación a evapotranspiración potencial significa un sobrante de agua durante ciertas épocas del año. Si se permitiera que el agua sobrante se escurriera por pendientes mayores del $25 \%$, ocurriría erosión por efecto del agua y esto generalmente estaría intensificado con usos de tierras que perturbarían la cobertura vegetativa.

B4: Precipitación anual media $<1500 \mathrm{~mm} ;$ P/ETP 1,0+; $<50 \%$ de arena; pendiente $<25 \%$ Las características de estas áreas son similares a las descritas en (B.1), (B.2) y (B.3): temperaturas frías y ubicación a elevadas alturas. Por lo tanto, los aniegos podrían ser un peligro en las áreas más planas. La agricultura es difícil debido a las cortas estaciones de crecimiento, aunque pueda ser posible el pastoreo de ganado. En algunas áreas, el pisoteo del ganado podría agravar los problemas de aniegos. Estas áreas usualmente tienen alta velocidad de vientos durante ciertas épocas del año. En consecuencia, podría ser necesario proteger artificialmente los suelos.

M1: Precipitación anual media $<1500 \mathrm{~mm} ; \mathrm{P} / \mathrm{ETP}=0,75-0,99 ;>50 \%$ de arena; pendiente $>25 \%$. Estas áreas normalmente se asocian con los procesos de desertificación. Ciertas combinaciones de estas características (muy baja precipitación y una relación P/ETP cerca de 0,76) presentan condiciones extremadamente propicias para el inicio del proceso de desertificación. En las áreas que reciben precipitación en el extremo alto del rango y con alta intensidad, la erosión de la superficie es una amenaza potencial. En las áreas más secas, el peligro de desertificación también puede ser ocasionado por el viento.

M2: Precipitación anual media $<1500 \mathrm{~mm} ; \mathrm{P} / \mathrm{ETP}=0,75-0,99 ;>50 \%$ de arena; pendiente $<25 \%$. La diferencia significativa entre áreas que tienen estas características y las áreas descritas en (M.1) arriba es el grado de la pendiente. En consecuencia, las áreas que corresponden a la descripción anterior tienen problemas de erosión de agua y viento, mientras que las áreas aquí descritas tienen problemas de salinidad de superficie, en particular aquellas áreas que tienen niveles de precipitación y relación P/ETP en el extremo bajo de los rangos indicados.

M3: Precipitación anual media $<1500 \mathrm{~mm} ; \mathrm{P} / \mathrm{ETP}=0,75-0,99 ;<50 \%$ de arena; pendiente $>25 \%$. Las áreas que tienen estas características están relativamente sujetas a que se inicie el proceso de desertificación. Bajo condiciones de menor precipitación y consiguiente escasez de vegetación, los 
eventos de intensas precipitaciones causan erosión del agua, y tal erosión es más severa si la superficie del suelo ha sido perturbada por pisoteo, arado, incendios o movimiento de suelos para propósitos de construcción. Asimismo, ha de ocurrir erosión si el suelo está descubierto durante períodos de sequías y vientos como frecuentemente lo está.

M4: Precipitación anual media $<1500 \mathrm{~mm} ; \mathrm{P} / \mathrm{ETP}=0,75-0,99 ;<50 \%$ de arena; pendiente $<25 \%$. Debido a la menor pendiente, los problemas de desertificación son: (1) erosión del viento, según como coincidan las épocas de sequía con aquellas de menor cobertura vegetativa, debido a actividades humanas (prácticas de cultivos) o temperaturas bajas; y (2) la salinidad de los suelos en áreas que tienen menos precipitación y mayor potencial de evapotranspiración.

A1: Precipitación anual media $<1500 \mathrm{~mm} ; \mathrm{P} / \mathrm{ETP}=0,01-0,75 ;>50 \%$ de arena; pendiente $>25 \%$. Estas características describen áreas que tienen un alto potencial para la desertificación. El proceso de desertificación se inicia fácilmente en estas áreas debido a la baja precipitación, el alto potencial de evapotranspiración, la rápida infiltración, y el aún más rápido escurrimiento de la precipitación que permanece en superficie debido a las pronunciadas pendientes. Aún la irrigación es potencialmente dañina, (1) debido a la cantidad de agua que probablemente se necesitará y la salinidad de sus residuos; y (2) ocasionado por la erosividad de los suelos arenosos en pendientes pronunciadas, aun cuando se use moderna tecnología de riego. Los ángulos de pendientes y la orientación son importantes por el hecho de que las pendientes que dan cara al sol serán mucho más secas y más calientes, excepto en latitudes muy frías, tendrán mucho menos crecimiento vegetal.

A2: Precipitación anual media $<1500 \mathrm{~mm} ; \mathrm{P} / \mathrm{ETP}=0,01-0,75 ;>50 \%$ de arena; pendiente $<25 \%$. Las condiciones en estas áreas son similares a las que se encuentran en las áreas descritas arriba, excepto que aquí las pendientes son mucho menos pronunciadas. El paisaje generalmente está menos definido geomorfológicamente. Como consecuencia, estas áreas más planas pueden tener un mayor nivel de humedad de suelos, porque reciben las descargas de las pendientes más arriba y porque la insolación generalmente será menos directa. La erosión de los vientos y la formación de dunas de arena podrían ser un problema si el suelo estuviera sin cobertura durante las épocas de viento y poca o nula precipitación. La salinidad de los suelos podría ser un problema, aunque el suelo más arenoso permite una rápida infiltración que lavaría las sales a los niveles inferiores en el perfil del suelo, donde formarían una capa de caliche.

A3: Precipitación anual media $<1500 \mathrm{~mm} ; \mathrm{P} / \mathrm{ETP}=0,01-0,75 ;<50 \%$ de arena; pendiente $>25 \%$ 
Determinación de zonas susceptibles al fenómeno de desertificación en el cantón Ibarra, provincia de Imbabura

La principal diferencia en las características de estas áreas respecto a las descritas en (A.2) es la cantidad de arena en el suelo. El menor porcentaje de arena en un suelo significa que los porcentajes de arcilla y de limo son mayores. Por otro lado, si los suelos son predominantemente arcillosos, aumenta el escurrimiento de la precipitación debido a la menor velocidad de infiltración. En consecuencia, las áreas aquí descritas pueden ser más secas que las que se describen en (A.2). Adicionalmente, la penetración de raíces puede ser reducida debido a que mayor porcentaje de arcilla, generalmente significa que el suelo es mucho más duro y menos desmenuzable bajo condiciones secas. A4: Precipitación anual media $<1500 \mathrm{~mm} ; \mathrm{P} / \mathrm{ETP}=0,01-0,75 ;<50 \%$ de arena; pendiente $<25 \%$.

Las características de esta área son muy similares a las presentadas en (A.3), excepto que las pendientes son mucho menos pronunciadas. Dado el mayor contenido de arcilla y pendientes menos pronunciadas, también podrían estar presentes niveles altos de alcalinidad del suelo y estos deben ser considerados al proponer un uso más intensivo del terreno, especialmente la agricultura con riego. Los problemas comunes de la desertificación son todos un potencial en estas áreas: aniegos (con irrigación); erosión del agua (especialmente a lo largo de los bordes de los cursos de aguas intermitentes); erosión del viento; formación de dunas y desplazamiento; y una reducida humedad efectiva.

\section{Resultados y Discusión}

\section{Precipitación $(P)$}

Se identificaron precipitaciones que van desde los 300 hasta los $3500 \mathrm{~mm}$ anuales (Cuadro 4). Estos resultados concuerdan con Bastidas (2004), que reportó precipitaciones promedio de $1027 \mathrm{~mm}$ en el cantón. El 79 \% de la superficie del cantón, presentan precipitaciones inferiores a los $1500 \mathrm{~mm} / \mathrm{año}$, volviéndose propensos al proceso de desertificación, mientras que solo el $21 \%$ muestran precipitaciones que limitan o no permiten el desarrollo de la desertificación, como es el caso de la parte noroeste del cantón (parroquia Lita), donde las lluvias van de 1700 a $3500 \mathrm{~mm}$. 
Cuadro 4. Precipitación en el cantón Ibarra

\begin{tabular}{|c|c|r|r|}
\hline \multirow{2}{*}{$\begin{array}{c}\text { Cantidad de agua precipitada } \\
(\mathbf{m m})\end{array}$} & Susceptibilidad & \multicolumn{3}{|c|}{$\begin{array}{c}\text { Superficie } \\
\text { cubierta/afectada }\end{array}$} \\
\cline { 3 - 4 } & & \multicolumn{1}{|c|}{ ha } & $(\mathbf{\%})$ \\
\hline $300-650$ & Alta & 24101,74 & 22 \\
\hline $550-950$ & Media & 14043,80 & 13 \\
\hline $750-1500$ & Baja & 48226,79 & 44 \\
\hline $1450-3500$ & Nula & 23332,96 & 21 \\
\hline Total & & 109705,29 & 100 \\
\hline
\end{tabular}

Fuente: Elaboración propia

\section{Evapotranspiración (ETP)}

Los valores registrados de ETP van de 625 a $1125 \mathrm{~mm}$. El 80 \% del área en estudio muestra una pérdida de agua inferior a los $900 \mathrm{~mm}$, quizá, esto se deba, a que la cantidad de agua que ingresa es menor a los $1500 \mathrm{~mm}$, y/o que la cobertura vegetal y uso de la tierra es escaza. El $20 \%$ restante, evidencia una evapotranspiración potencial superior a los $900 \mathrm{~mm}$, correspondiendo a zonas con mayor cobertura de vegetación natural (zona Lita) y por ende mayor evaporación y transpiración de la misma.

\section{Relación P/ETP}

En base a los cálculos realizados (Cuadro 5) se evidenció que, a menor valor de relación P/ETP mayor es la posibilidad de desarrollarse el fenómeno de la desertificación, probablemente, debido a que la cantidad de agua que ingresa es menor a la cantidad de agua que se pierde a través de la evaporación y transpiración.

Cuadro 5. Relación Precipitación/Evapotranspiración en el cantón Ibarra

\begin{tabular}{|c|c|}
\hline $\begin{array}{c}\text { Relación P/ETP } \\
\text { (promedio) }\end{array}$ & Susceptibilidad \\
\hline 0,54 & Alta \\
\hline 0,87 & Media \\
\hline 1,5 & Baja \\
\hline 2,22 & Nula \\
\hline
\end{tabular}

Fuente: Elaboración propia 
Determinación de zonas susceptibles al fenómeno de desertificación en el cantón Ibarra, provincia de Imbabura

Mientras que, a mayor relación, la susceptibilidad a la desertificación es menor. De acuerdo con el Atlas Mundial de Desertificación (UNEP, 1992), las tierras áridas tienen una relación de precipitación anual promedio (P) respecto a la evapotranspiración potencial (ETP) menor de 0,65.

Pendiente de la forma del relieve

El Cuadro 6, nos indica, en porcentaje, la superficie que ocupa cada rango de pendiente, podemos observar que más del $60 \%$ del área cantonal, presenta pendientes fuertes a muy fuertes (40 a 100 \%),lo cual influye en la velocidad y la cantidad de la corriente de agua en superficie, así como, en la cantidad e intensidad de luz solar que recibe un determinado sitio (OEA, 1993), incurriendo junto a otras variables: precipitación y textura- en el desarrollo del fenómeno.

Cuadro 6. Pendiente de las formas del relieve existentes en el cantón Ibarra

\begin{tabular}{|c|r|}
\hline Pendiente & Superficie ocupada (\%) \\
\hline 70 a $100 \%$ & 50,10 \\
\hline 40 a $70 \%$ & 10,40 \\
\hline 5 a $12 \%$ & 9,80 \\
\hline 100 a $150 \%$ & 8,20 \\
\hline 12 a $25 \%$ & 7,00 \\
\hline 2 a $5 \%$ & 6,30 \\
\hline 25 a $40 \%$ & 5,70 \\
\hline 150 a $200 \%$ & 0,70 \\
\hline 0 a $2 \%$ & 0,10 \\
\hline
\end{tabular}

Fuente: Elaboración propia

\section{Clase de textura de suelo}

Mediante los análisis físicos (campo) y químicos (laboratorio) realizados a los perfiles de suelo generados en el cantón, se determinaron nueve (9) tipos o clases de texturas de suelo (Cuadro 7), donde el porcentaje de arena, es indispensable para determinar la desertificación.

La textura predominante en el cantón Ibarra es de tipo franca, con el 48 \%, donde la cantidad de arena es del $40 \%$ y de acuerdo a la FAO (2009) es una cantidad óptima de arena, es decir, la cantidad de agua que se infiltra, no es mayor a la que se retiene en los espacios porosos. Esta información es importante ya que influye en características como la humedad y fertilidad del suelo, las cuales, a su vez, contribuyen en el proceso de erosión y desertificación. 
Cuadro 7. Clase de textura de suelo en el cantón Ibarra

\begin{tabular}{|l|c|r|}
\hline Tipo de textura de suelo & Cantidad de Arena (\%) & Superficie ocupada (\%) \\
\hline Franco & 40 & 48,8 \\
\hline Franco arcilloso & 35 & 18,6 \\
\hline Franco arenoso & 65 & 15,3 \\
\hline Franco arcillo-arenoso & 35 & 8,6 \\
\hline Arcillo-arenoso & 55 & 2,1 \\
\hline Areno francoso & 80 & 1,4 \\
\hline Arcilloso & 10 & 1,4 \\
\hline Franco limoso & 20 & 0,8 \\
\hline Arena & 90 & 0,002 \\
\hline
\end{tabular}

Fuente: Elaboración propia

\section{Susceptibilidad al fenómeno de la desertificación}

Tomando en cuenta las variables anteriores (precipitación, evapotranspiración, relación P/ETP, pendiente de la forma del relieve y la clase textural) y su interacción, se identificó en el cantón Ibarra las zonas susceptibles al fenómeno de la desertificación mediante cuatro niveles o grados: nula, baja, media y alta (Figura 2).

De acuerdo al Cuadro 8, el $35 \%$ de la superficie total del cantón presenta una susceptibilidad media (13\%) a alta (2\%) al fenómeno de la desertificación, en tanto que, el $44 \%$ y $21 \%$ presentan baja y nula susceptibilidad al desarrollo de la desertificación, respectivamente.

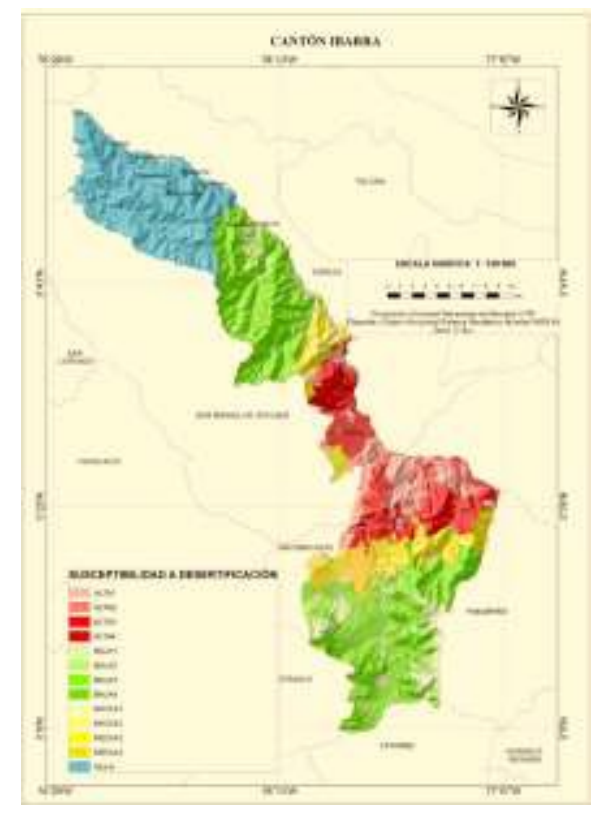

Figura 2.Mapa de Zonas Susceptibles al Fenómeno de la Desertificación

Fuente: Elaboración propia 
Determinación de zonas susceptibles al fenómeno de desertificación en el cantón Ibarra, provincia de Imbabura

Cuadro 8. Susceptibilidad a desertificación en el cantón Ibarra

\begin{tabular}{|c|r|r|}
\hline \multirow{2}{*}{ Zonas de susceptibilidad } & \multicolumn{2}{|c|}{ Superficie afectada } \\
\cline { 2 - 3 } & (ha) & \multicolumn{1}{c|}{ (\%) } \\
\hline Alta & 24101,74 & 22 \\
\hline Media & 14043,80 & 13 \\
\hline Baja & 48226,79 & 44 \\
\hline Nula & 23332,96 & 21 \\
\hline TOTAL & 109705,29 & 100 \\
\hline
\end{tabular}

Fuente: Elaboración propia

\section{Descripción de la susceptibilidad al fenómeno de la desertificación}

\section{Zonas de susceptibilidad nula}

Esta zona se localiza al noroeste del cantón, ocupa una superficie de 23 332,96 ha, cuya zona de vida según Holdridge (1967) corresponde al bosque húmedo, con precipitaciones que superan los 1500 $\mathrm{mm}$, incluso llegando hasta los $3500 \mathrm{~mm}$ medias anuales, esta variable es determinante para calificar el grado de susceptibilidad, siempre y cuando exista una cobertura y protección del suelo, para evitar que se produzca una pérdida de suelo por erosión hídrica, fenómeno que puede presentarse en lugares donde las precipitaciones son fuertes y no consecutivas.

\section{Zonas de susceptibilidad baja}

Se encuentra ubicada en la parte sur de Ibarra, principalmente, en los sectores de Angochagua y La Esperanza, y en parte de las parroquias Ibarra y Ambuquí, cubre una superficie de 48226,79 ha. Esta zona presenta precipitaciones que van de 700 a $1500 \mathrm{~mm}$ medias anuales y una relación de P/ETP > 1, es decir, la precipitación es mayor a la evapotranspiración potencial, probablemente debido a la falta de cobertura y uso del suelo. Por otra parte, cuando la pendiente es superior al $25 \%$ se podría producir una erosión hídrica laminar en el suelo, ya que el agua de lluvia por el grado de la pendiente no se infiltra, si no, que circula sobre la superficie del terreno, arrastrando la capa superficial del suelo. Sin embargo, bajo estas condiciones hay una reducida probabilidad para el desarrollo de la desertificación.

\section{Zonas de susceptibilidad media}

Esta zona ocupa un área de 14043,80 ha, se presenta en la parte sur de la parroquia Carolina y en la parte central de Ambuquí e Ibarra, donde se registra, en promedio, precipitaciones de $750 \mathrm{~mm}$ y una evapotranspiración de $850 \mathrm{~mm}$, es decir, existe una mayor cantidad de agua evaporada y transpirada en comparación con la precipitada, dando como resultado una mayor susceptibilidad a que inicie el 
proceso de desertificación, en especial, en las zonas cuyos suelos son arenosos y sus pendientes son fuertes (> $25 \%$ ), condiciones aptas para que pueda ocurrir una erosión del suelo por acción del agua, y más aún si no existe cobertura vegetal de la tierra y el suelo ha sufrido algún tipo de perturbación, como por ejemplo, pisoteo del ganado, arado, tala y quema de la vegetación, entre otros. Además hay que tener en cuenta, que las precipitaciones empiezan a ser escazas por lo que se debería aplicar mecanismos de regadío, constituyendo en un gasto para los agricultores o productores del sector agrícola.

\section{Zonas de susceptibilidad alta}

Esta zona de susceptibilidad afecta a una superficie de 24101,74 ha. Se ubica en la parte central del cantón, principalmente, en Salinas de Ibarra, con precipitaciones que van de 300 a $700 \mathrm{~mm}$, una evapotranspiración potencial de 725 a $975 \mathrm{~mm}$, y con una relación de precipitación/ evapotranspiración potencial de 0,3 a 0,7 ; todas estas características son óptimas para que se inicie fácilmente el proceso de desertificación, y si a éstas variables se le suma la escaza vegetación y la actividad humana (quema, tala, sobrepastoreo, etc) se agudiza el fenómeno. Se debe considerar, al igual que en el caso anterior, que las precipitaciones son mínimas o escazas, y que es necesario implementar mecanismos de riego, para poder desarrollar procesos de producción agrícola o de prácticas de cobertura de la tierra. Así mismo, los bajos niveles de precipitación, provocan un incremento en el pH del suelo, dando como resultado la acumulación, sobre todo, de carbonato de sodio, provocando la impermeabilidad de los suelos, lo cual dificulta la infiltración del agua y ayuda a la erosión del mismo.

\section{Conclusiones}

Se obtuvo 4 categorías de susceptibilidad a desertificación: alta, media, baja y nula, siendo la tercera la de mayor superficie de ocupación en la zona de estudio. Las zonas se distribuyen en la parte sur del área en estudio, principalmente, en las parroquias de Angochagua y La Esperanza, y cuya característica principal es relación de $\mathrm{P} / \mathrm{ETP}>1$, como consecuencia de precipitaciones que van de 700 a $1500 \mathrm{~mm}$ medias anuales.

-La zona con susceptibilidad alta está localizada en la parte central del cantón, principalmente, en Salinas de Ibarra, en áreas semiáridas, con precipitaciones que van de 300 a $700 \mathrm{~mm}$, y una evapotranspiración potencial de 725 a $975 \mathrm{~mm}$, es decir, la cantidad de agua que sale supera a la cantidad de agua que ingresa. Además, la baja cantidad de agua de lluvia, conlleva a un incremento 
Determinación de zonas susceptibles al fenómeno de desertificación en el cantón Ibarra, provincia de Imbabura

en el pH del suelo, dando como resultado la acumulación de carbonato de sodio, provocando suelos con una fertilidad baja.

-La zona de susceptibilidad nula que se observa en el cantón, se localiza, en su mayoría, en el sector de Lita, donde la zona de vida corresponde al bosque húmedo y las precipitaciones medias anuales son mayores a los $1500 \mathrm{~mm}$. Por otra parte, hay que tomar en cuenta la cobertura y protección del suelo, ya que si éste se encuentra descubierto podría presentarse la amenaza de erosión por acción del agua.

La zona de susceptibilidad media se encuentra presente en la parte sur de la parroquia La Carolina y en la parte central de Ambuquí e Ibarra, y al igual que la zona con susceptibilidad alta, la cantidad de agua que se evapora es mayor a la cantidad de agua que se precipita, donde cuyas precipitaciones no superan los $750 \mathrm{~mm}$ en promedio anual. Se debe tener en cuenta, que las precipitaciones empiezan a ser escazas por lo que es necesario aplicar mecanismos de regadío para realizar actividades agrícolas, constituyendo en un gasto para los agricultores o productores del sector.

\section{Referencias Bibliográficas}

Abraham, E.; Montana, E.; Torres, L. (2006). Desertificación e Indicadores: Posibilidades de Medición integrada de fenómenos complejos. Revista Electrónica de Geografía y Ciencias Sociales ISSN: 1138-9788. Universidad de Barcelona. Vol. x, 24.

Bastidas, S.; CASCO, C. (2004). Evaluación de Alternativas Metodológicas para el Manejo Integrado de Sueloen el Cultivo de Papa (Solonum Tuberosum L) en la provincia de Carchi. Tesis. Ing. Agrp. Ibarra. Pontificia Universidad Católica del Ecuador. p. 55

COP6. (2003). Informe central. Sexta Conferencia de las Partes. Convención de las Naciones Unidas de Lucha Contra la Desertificación y la Sequía. Cuba.

FAO. (1993). Desarrollo sostenible de tierras áridas y lucha contra la desertificación. Organización de las Naciones Unidas para la Agricultura y la Alimentación. Recuperado de $<$ https://bit.ly/2ZUgmhE $>$ Consultado el: 16 de enero de 2019.

FAO. (2009). Guía para la descripción de suelos. Cuarta edición. Traducido y adaptado al castellano por Ronald Vargas Rojas. Organización de las Naciones Unidas para la Agricultura y la Alimentación. (Proyecto FAOSWALIM, Nairobi, Kenya-Universidad Mayor de San Simón, Bolivia). Roma, Italia. 
GAD Ibarra. (2016). Plan de desarrollo y ordenamiento territorial del cantón Ibarra (PDOT). Gobierno Autónomo Descentralizado Municipal de Ibarra. Recuperado de $<$ https://bit.ly/2Y3jyWO> Consultado el: 17 de enero de 2019.

García, M.; González, J.; Ibáñez, J.; López, P.; Martín, M.; Puigdefábregas, J.; De La Rosa, D.; Rubio, L. (1996). Programa Interáreas del CSIC sobre Desertificación en ambientes mediterráneos: Aspectos físicos, culturales, sociales y económicos. Instituto Pirenaico de Ecología. CSIC. Zaragoza: 27 pp.

Holdridge, R. (1967). Life Zone Ecology. San José, Costa Rica: Tropical Science Center.

IEE y MAGAP. (2015). Zonas de Susceptibilidad a Desertificación en el Ecuador Continental

Escala 1:50 000. Instituto Espacial Ecuatoriano - Ministerio de Agricultura Ganadería Acuacultura y Pesca. Recuperado de

INAMHI. (2017). Base Nacional de Datos Hidrometeorológicos. Gestión de Información Hidrometeorológica. Dirección de Información Hidrológica. Quito. Instituto Nacional de Meteorología e Hidrología.

López, F. (2001). El riesgo de desertificación. En F. Martín de Santa Olalla Mañas (ed.). Agricultura y desertificación. pp. 15-38. Ediciones Mundi-Prensa. Madrid, España.

López, F. (2004). Las tierras mediterráneas en la frontera de la desertificación. Métodos y planes de acción para combatir el proceso de degradación. En: Revista Aranzadi de derecho Ambiental. Actas del V Congreso Nacional de Derecho Ambiental. Editorial Aranzadi S.A. 1-30 pp.

MAE y MAG (2017). Lucha contra la desertificación. Ministerio del Ambiente Ecuatoriano Ministerio de Agricultura y Ganadería. Recuperado de http://www.ambiente.gob.ec/ecuadorluchar-contra-la-desertificacion/ Consultado el: 17 de enero de 2019.

OEA. (1993). Manual Sobre el Manejo de Peligros Naturales en la Planificación para el Desarrollo Regional. Organización de los Estados Americanos. Estados Unidos, Washington, D.C. Recuperado de < https://bit.ly/2JEbeGY> Consultado el: 13/02/2019.

Oba, G. 2001. The effect of multiple droughts on cattle in Obbu, Northen Kenya. Journal of Arid Environments.

Oropeza. (2007). Evaluación de la vulnerabilidad a la desertificación. Universidad Nacional Autónoma de México. Recuperado de https://bit.ly/2UN5kYd Consultado el: 25/02/2019.

Paruelo, M., Aguiar, M. (2003). El impacto humano sobre los ecosistemas: el caso de la desertificación. Ciencia Hoy 13:48-59. 
Determinación de zonas susceptibles al fenómeno de desertificación en el cantón Ibarra, provincia de Imbabura

PNUMA. (1991). Estado de la desertificación y aplicación del Plan de Acción de las Naciones Unidas para combatir la desertificación. Informe del Director Ejecutivo. Nairobi, UNEP, GCSS.

PNUMA. (2012). Perspectivas del Medio Ambiente Mundial. GEO 5. Recuperado de http://www.unep.org/spanish/geo/geo5.asp, verificado 7 de junio de 2012. Consultado el: 21 de enero de 2019.

Pulido, G. y López, A. (2007). IV Foro de Investigaciones por la Conservación y II Simposio de Áreas Naturales Protegidas del Estado de Hidalgo. Universidad Autónoma de Hidalgo, México.

Ruiz, T.; Febles, G (2004). La desertificación y la sequía en el mundo. Revista Electrónica de Avances en Investigación Agropecuaria ISSN: 0188-7890. Universidad de Colima México .Vol 8. x, 2.

Salomón, M., Guamán, C., Rubio, C., Galárraga, R., Abraham, E. (2008). Indicadores de uso del agua en una zona de los Andes centrales de Ecuador. Estudio de la cuenca del Río Ambato. Recuperado de < https://bit.ly/2GW3pMU> Consultado el: 22 de enero de 2019.

SENPLADES. (2013). Plan Nacional para el Buen Vivir 2013 - 2017. Secretaría Nacional de Planificación y Desarrollo. Quito, Pichincha, Ecuador.

UNEP. (1992). Status of desertification and implementation of the United Nations Plan of Action to Combat Desertification. Programa Ambiental de las Naciones Unidas. USA: United Nations Environment Programme.

UNITED NATIONS. (2018). Día mundial de lucha contra la desertificación. Recuperado de $<$ www.unccd.int $>$ Consultado el: 17/07/2018.

USDA. (2006). Claves para la Taxonomía de Suelos. United States department of Agriculture. Décima Edición. EE. UU.

Valderrama, J. (2017). Los desiertos y la desertificación. Editorial Catarata, México, C1.

Valverde, J. (2007). Riego y Drenaje. Editorial Universidad Estatal a Distancia. ISBN: 9977-64-9944 Recuperado de <https://bit.ly/2ZZY2Ea> Consultado el: 25/02/2019. 